\begin{tabular}{|c|c|}
\hline$\beth$ & $\begin{array}{c}\text { International Journal of Current Research } \\
\text { and Academic Review }\end{array}$ \\
\hline $\begin{array}{l}\text { YCELLENT } \\
\text { BLLISHERS }\end{array}$ & $\begin{array}{r}\text { ISSN: } 2347-3215 \text { (Online);,; Volume 5;,; } \\
\text { Journal homepage: http://www }\end{array}$ \\
\hline
\end{tabular}

doi: http://dx.doi.org/10.20546/ijcrar.2017.501.006

\title{
Comparative Analysis of Assessment of RV Dysfunction using 2D FAC and TAPSE in Patients of COPD and their Correlation with Pulmonary Function Test (PFT) and 6 Minute Walk Test (6MWT)
}

\author{
Manish Kumar Bansal ${ }^{1}$ and Bechan Kumar Gautam ${ }^{2 *}$ \\ ${ }^{l}$ M.D. ${ }^{2}$ Medicine, Junior resident, Professor, P.G. Department of Medicine, Sarojini Naidu Medical College, \\ Agra, India \\ *Corresponding author
}

\begin{abstract}
COPD is associated with significant extra-pulmonary (systemic) effects among which cardiac complications are most common. COPD affects primarily the right side of heart and cor pulmonale is one of the important causes of increased morbidity and mortality in patients of COPD. Echocardiography provides a rapid, non-invasive method to evaluate the cardiac changes. The aim of the study was to comparatively analyse the 2D FAC and TAPSE as a marker of RV dysfunction in patients of COPD and correlating them with PFT and 6MWT. A total of 100 COPD patients admitted in the P.G. department of Medicine S.N. Medical College, Agra between March 2015 and June 2016 were subjected to thorough clinical evaluation, spirometry, 6MWT and 2D echocardiography and the results were analysed to establish comparison between 2D FAC and TAPSE and their correlation with PFT and 6MWT. In our study the values of both 2D FAC and TAPSE correlated positively with the value of FEV1 (PFT) and 6MWT i.e. lower value of 2D FAC and TAPSE corresponded to lower value of FEV1 (higher GOLD stage) and 6MWT indicating a poorer prognosis in patients of COPD. As per 2D FAC 30\% of the patients had normal RV function, $70 \%$ had RV dysfunction and as per TAPSE $19 \%$ patients had normal RV function, $81 \%$ had RV dysfunction. Among patients with RV systolic dysfunction, as per the values of 2D FAC, 29\% patients were with mild systolic dysfunction, $26 \%$ patients were with moderate RV systolic dysfunction and $15 \%$ were with severe RV systolic dysfunction and as per the values of TAPSE , $40 \%$ patients were with mild systolic dysfunction, $26 \%$ patients were with moderate RV systolic dysfunction and $15 \%$ were with severe RV systolic dysfunction showing that TAPSE was a more sensitive indicator of mild RV dysfunction while for diagnosing moderate and severe RV dysfunction both parameters had similar sensitivity. In present study TAPSE picked $11 \%$ cases of mild RV dysfunction which were negative by 2D FAC. So TAPSE should be preferred over 2D FAC in assessment of RV function especially in early stages of COPD.
\end{abstract}

\section{Article Info}

Accepted: 05 January 2016

Available Online: 20 January 2017

\section{Keywords}

COPD, Cardiac complications, PFT, Echocardiography, 2D FAC,

TAPSE, 6MWT. 


\section{Introduction}

Chronic Obstructive Pulmonary Disease (COPD) has been defined by the Global Initiative for Chronic Obstructive Lung Disease (GOLD) as a disease state characterized by persistent airflow limitation that is usually progressive and associated with an enhanced chronic inflammatory response in the airways and lungs to noxious particles or gases (Global initiative for chronic obstructive lung disease).

Spirometry is the key test necessary for diagnosis of COPD.

COPD is the fourth leading cause of death and affects $>16$ million persons in the United States. COPD is also a disease of increasing public health importance around the world. GOLD estimates suggest that COPD will rise from the sixth to the third most common cause of death worldwide by 2020 (John).

In India, COPD is 2nd most common lung disorder after pulmonary tuberculosis. The disease is frequently encountered in the middle aged patients and is rare below age of 35 . It affects male more than female. It is equally prevalent in rural and urban areas (Surendra).

COPD is associated with a decline in the functional exercise capacity of the patient.

Six Minute Walk Test (6MWT) is a practical simple test for the objective evaluation functional exercise capacity (ATS statement, 2002). This test measures the distance that a patient can quickly walk on a flat, hard surface in a period of 6 minutes (the 6MWD).

COPD is associated with significant extra-pulmonary (systemic) effects among which cardiac complications are most common. Cardiovascular disease accounts for approximately $50 \%$ of all hospitalization and nearly onethird of all deaths, in cases where (FEV1) is $50 \%$ of predicted. So, a timely prediction about the cardiac involvement is of immense importance.

COPD affects primarily the right side of heart (Gupta et $a l ., 2011)$. Right Ventricular dysfunction and dilation are correlated to limited exercise capacity and poor outcome but often neglected in the clinical settings (Di salvo et al., 1995; Gavazzi et al., 1997).
Echocardiography provides a rapid, non-invasive and accurate method to evaluate the RV functions along with left ventricular function.

The purpose of our study was to evaluate the RV functions in patients of COPD by using 2D FAC and TAPSE with an aim to find a simple way of predicting cardiac morbidity in COPD and their correlation with PFT and 6MWT.

\section{Materials and Methods}

This cross sectional observational study was conducted on 100 patients of COPD over a period of 15 month (March 2015 to June 2016) in P.G department of medicine Sarojini Naidu Medical College, Agra.

Patients who were fulfilling all the inclusion criteria and gave informed consent were included in the study.

\section{Inclusion Criteria}

1. Clinical criteria - Patients with symptoms and signs of COPD

2. Spirometry criteria - On spirometry FEV1/FVC $<0.7$ after use of bronchodilator

\section{Exclusion Criteria}

1. Patients with other respiratory or cardiac problems (like bronchial asthma, pulmonary tuberculosis (TB), lung malignancy, lung fibrosis or collapse, bronchiectasis, pleural effusion, pleural tumours, primary pulmonary hypertension, valvular heart disease, congenital heart disease, pericardial disease, systemic hypertension, coronary artery disease, changes suggestive of my ischemia on electrocardiography, RWMA or global hypokinesia on echocardiography, cardiomyopathy).

\section{Poor echo window.}

\section{Inability to perform PFT.}

Methodology : First all the patients presenting with symptoms and signs consistent with COPD were subjected to thorough clinical evaluation then the diagnosis of COPD was confirmed and evaluated for staging; by PFT (according to GOLD criteria) in the post graduate Department of Medicine, Sarojini Naidu Medical College, Agra, India. Subsequently, all the 
patients underwent the 6MWT and 2D echocardiography in the post graduate Department of Medicine of Sarojini Naidu Medical College, Agra, with special emphasis on the function of the right heart.

All the patients were investigated by spirometry. The PFTs parameters included were FEV1, FVC, FEV1/FVC ratio (American Thoracic Society, 1995). The results of the PFTs were recorded in percent of predicted values.

All the patients underwent 6MWT over a walking course of $30 \mathrm{~m}$ in length marked by 2 orange cones.

Thereafter all the patients were subjected to echocardiography. The echocardiography parameters were2D FAC and TAPSE.

All the data collected were analysed by SSPS version-17 for mean and SD and suitable statistical test were applied wherever needed.

\section{Results and Discussion}

Two dimensional fractional area change represents a "surrogate" measurement of RV EF and is expressed as a percentage change in the RV chamber area from enddiastole to end-systole, rather than changes in volume.

In a study by Anavekar et al., (2007) RV FAC was found to correlate best with CMR-derived RV EF (CMR vs RV FAC, $\mathrm{r}=0.80, \mathrm{P}<.0001 ; \mathrm{CMR}$ vs transverse fractional shortening, $\mathrm{r}=0.12, \mathrm{P}=.48 ; \mathrm{CMR}$ vs TAM, $\mathrm{r}=0.17, \mathrm{P}$ $=.30)$.

TAPSE as a measure of RV ejection fraction was first proposed by Kaul et al., in 1984, who demonstrated a close to RV ejection fraction determined by radionuclide techniques. This has been confirmed in subsequent studies in patients with HF using thermo-dilution techniques or magnetic resonance imaging.

The close correlation of TAPSE to RV ejection fraction measured by thermo-dilution or radionuclide techniques has also been established in the lower range of ejection fraction (Brieke, 2005; Anconina et al., 1993).

In COPD right heart failure occurs due to development of pulmonary hypertension and since RV dysfunction bears a prognostic value in patients of COPD, we studied RV dysfunction in them using the following two echo parameters, the 2D FAC and the TAPSE and comparatively analysed them as which among them is a better marker for the assessment of RV dysfunction in patients of COPD. We also tried to find out any correlation between them and with PFT and 6MWT. The present study was conducted over a period of 15 months in P. G. Department of Medicine, Sarojini Naidu Medical College, Agra, Uttar Pradesh, India.

Table no. 1 shows that $21 \%$ cases belonged to GOLD stage-I, $38 \%$ belonged to GOLD stage-II, $26 \%$ belonged to GOLD stage-III, and $15 \%$ belonged to GOLD stageIV.

Table no. 2 shows that, 2D RV FAC (\%) was decreasing as the GOLD stage was increasing. In GOLD stage-I the mean 2D RV FAC was $41.05 \%$, in GOLD stage-II it was $29.68 \%$, in GOLD stage-III it was $20.12 \%$ and in GOLD stage-IV it was $14.33 \%$ i.e a lower value of 2D FAC corresponded to a lower value of FEV1 (higher GOLD stage) and thus indicated poorer prognosis in patients of COPD. On applying ' $t$ ' test, the mean values of 2D FAC in various GOLD stages were found to be statistically significant and 'p' value came out to be $<0.0001$.

Maumita das et al., (2014) in their study "Assessment of RV Function in patients of COPD" concluded that FAC $\%$ and RIMP can be vital prognostic factors for RV function apart from SPAP and, TAPSE.

Table no. 3 shows that value of TAPSE (mm) was decreasing as the GOLD stage was increasing. In GOLD stage-I, the mean TAPSE was $17.43 \mathrm{~mm}$, in GOLD stageII it was $12.63 \mathrm{~mm}$, in GOLD stage-III it was $7.27 \mathrm{~mm}$ and in GOLD stage-IV it was $3.73 \mathrm{~mm}$ i.e a lower value of TAPSE corresponded to a lower value of FEV1 (higher GOLD stage) and thus indicated poorer prognosis in patients of COPD. On applying ' $t$ ' test, the mean values of TAPSE in various GOLD stages were found to be statistically significant and ' $p$ ' value came out to be $<0.0001$.

Chetan Rathi et al., (2015) conducted a study "Pulmonary hypertension in mild-moderate COPD : an early link"on 100 patients of COPD concluded that severity of COPD correlated very well with proportionate changes in RV function and PASP (pulmonary hypertension).

Table no. $4 \& 5$ indicates that as per the values of $2 \mathrm{D}$ FAC $30 \%$ patients had normal RV systolic function and $70 \%$ had RV systolic dysfunction and as per the values of TAPSE 19\% patients had no RV systolic dysfunction and $81 \%$ had RV systolic dysfunction. 
Among patients with RV systolic dysfunction, as per the values of 2D FAC, $29 \%$ patients were with mild systolic dysfunction, $26 \%$ patients were with moderate RV systolic dysfunction and $15 \%$ were with severe RV systolic dysfunction and as per the values of TAPSE, $40 \%$ patients were with mild systolic dysfunction, $26 \%$ patients were with moderate RV systolic dysfunction and $15 \%$ were with severe RV systolic dysfunction.

In table no. 4 taking GOLD staging and 2D FAC together into consideration in these patients, in GOLD stage-I $95.24 \%$ patients had no RV systolic dysfunction while the remaining $4.76 \%$ had RV systolic dysfunction (mild), in GOLD stage-II $26.32 \%$ had no RV systolic dysfunction and the remaining $73.68 \%$ had RV systolic dysfunction (mild), in GOLD stage-III $100 \%$ patients had RV dysfunction (moderate) and again in GOLD stage-IV 100\% had RV systolic dysfunction (severe).

In table no. 5 taking GOLD staging and TAPSE together into consideration in these patients, in GOLD stage-I 90.48\% patients had no RV systolic dysfunction while the remaining 9.52\% had RV systolic dysfunction (mild), in GOLD stage-II $100 \%$ had RV systolic dysfunction (mild), in GOLD stage-III $100 \%$ patients had RV dysfunction (moderate) and again in GOLD stage-IV $100 \%$ had RV systolic dysfunction (severe) [Table no.5].

Gupta, Ritesh Kumar Agrawal, Srivastav, Ved (2011), in their study of "Echocardiographic evaluation of heart in COPD patients and its correlation with severity of disease" in 40 patients, tricuspid regurgitation (TR) was observed in 27/40 cases (67.5\%). Pulmonary hypertension $(\mathrm{PH})$ was observed in 17/27 (63\%) cases in which prevalence of mild, moderate and severe $\mathrm{PH}$ were 10/17 (58.82\%), 4/17 (23.53\%), and 3/17 (17.65\%), respectively. The frequencies of $\mathrm{PH}$ gradually increases with increase in severity of COPD.

Table no. 6 shows that the mean distance walked upon by cases in 6MWT was decreasing as the GOLD stage was increasing, in stage-I the mean distance walked upon by cases was $431 \mathrm{~m}$, in stage-II it was $337.13 \mathrm{~m}$, in stage-III it was $222.15 \mathrm{~m}$ and in stage-IV it was $165 \mathrm{~m}$ and on applying ' $t$ ' test the mean values of 6MWT in various GOLD stages were found to be statistically significant and 'p' value came out to be $<0.0001$ [Table no.6].

Table.1 Distribution of cases according to GOLD staging of COPD

\begin{tabular}{|l|l|l|}
\hline GOLD stage & No. of cases & Percentage \\
\hline I & 21 & 21 \\
\hline II & 38 & 38 \\
\hline III & 26 & 26 \\
\hline IV & 15 & 15 \\
\hline Total & $\mathbf{1 0 0}$ & $\mathbf{1 0 0}$ \\
\hline
\end{tabular}

Table.2 Distribution of value of 2D FAC (\%) in different GOLD stages of COPD

\begin{tabular}{|l|l|l|l|}
\hline Stages & No. & Mean & SD \\
\hline Stage -I & 21 & 41.05 & 5.04 \\
\hline Stage -II & 38 & 29.68 & 2.14 \\
\hline Stage -III & 26 & 20.12 & 1.61 \\
\hline Stage -IV & 15 & 14.33 & 1.29 \\
\hline
\end{tabular}

Table.3 Distribution of value of TAPSE ( $\mathrm{mm}$ ) in different GOLD stages of COPD

\begin{tabular}{|l|l|l|l|}
\hline Stages & No. & Mean & SD \\
\hline Stage - I & 21 & 17.43 & 2.01 \\
\hline Stage -II & 38 & 12.63 & 1.10 \\
\hline Stage -III & 26 & 7.27 & 1.19 \\
\hline Stage -IV & 15 & 3.73 & 0.80 \\
\hline
\end{tabular}


Table.4 Prevalence and Grading of RV systolic dysfunction on the basis of 2D FAC (\%) in patients of COPD :

\begin{tabular}{|c|c|c|c|c|c|c|c|c|c|c|}
\hline \multirow{3}{*}{ Stages } & \multirow{2}{*}{\multicolumn{2}{|c|}{ Cases }} & \multicolumn{8}{|c|}{ 2D FAC $(\%)$} \\
\hline & & & \multicolumn{2}{|c|}{$\geq 35$ (Normal) } & \multicolumn{2}{|c|}{ 25-34 (Mild) } & \multicolumn{2}{|c|}{ 18-24 (Moderate) } & \multicolumn{2}{|c|}{$\leq 17$ (Severe) } \\
\hline & No. & $\%$ & No. & $\%$ & No. & $\%$ & No. & $\%$ & No. & $\%$ \\
\hline Stage $-\mathrm{I}$ & 21 & 100 & 20 & 95.24 & 1 & 4.76 & 0 & 0 & 0 & 0 \\
\hline Stage -II & 38 & 100 & 10 & 26.32 & 28 & 73.68 & 0 & 0 & 0 & 0 \\
\hline Stage -III & 26 & 100 & 0 & 0 & 0 & 0 & 26 & 100 & 0 & 0 \\
\hline Stage -IV & 15 & 100 & 0 & 0 & 0 & 0 & 0 & 0 & 15 & 100 \\
\hline Total & 100 & 100 & 30 & 30 & 29 & 29 & 26 & 26 & 15 & 15 \\
\hline
\end{tabular}

Table.5 Prevalence and Grading of RV systolic dysfunction on the basis of TAPSE ( $\mathrm{mm}$ ) in patients of COPD

\begin{tabular}{|c|c|c|c|c|c|c|c|c|c|c|}
\hline \multirow{3}{*}{ Stages } & \multirow{2}{*}{\multicolumn{2}{|c|}{ Cases }} & \multicolumn{8}{|c|}{ TAPSE (mm) } \\
\hline & & & \multicolumn{2}{|c|}{ 16-20 (Normal) } & \multicolumn{2}{|c|}{ 11-15 (Mild) } & \multicolumn{2}{|c|}{ 6-10 (Moderate) } & \multicolumn{2}{|c|}{$\leq 5$ (Severe) } \\
\hline & No. & $\%$ & No. & $\%$ & No. & $\%$ & No. & $\%$ & No. & $\%$ \\
\hline Stage $-\mathrm{I}$ & 21 & 100 & 19 & 90.48 & 2 & 9.52 & 0 & 0 & 0 & 0 \\
\hline Stage -II & 38 & 100 & 0 & 0 & 38 & 100 & 0 & 0 & 0 & 0 \\
\hline Stage -III & 26 & 100 & 0 & 0 & 0 & 0 & 26 & 100 & 0 & 0 \\
\hline Stage -IV & 15 & 100 & 0 & 0 & 0 & 0 & 0 & 0 & 15 & 100 \\
\hline Total & 100 & 100 & 19 & 19 & 40 & 40 & 26 & 26 & 15 & 15 \\
\hline
\end{tabular}

Table.6 Mean distances walked upon by cases in different GOLD stages of COPD during 6MWT

\begin{tabular}{|l|l|l|l|}
\hline Stages & No. & Mean & SD \\
\hline Stage -I & 21 & 431 & 33.9 \\
\hline Stage -II & 38 & 337.13 & 20.3 \\
\hline Stage -III & 26 & 222.15 & 18.21 \\
\hline Stage -IV & 15 & 165 & 8.99 \\
\hline
\end{tabular}

Table.7 Relation between 6MWT (m) and 2D FAC (\%)

\begin{tabular}{|l|l|l|}
\hline Stages & 6MWT (m) & 2D FAC (\%) \\
\hline Stage-I & 431 & 41.05 \\
\hline Stage-II & 337.13 & 29.68 \\
\hline Stage-III & 222.15 & 20.12 \\
\hline Stage-IV & 165 & 14.33 \\
\hline
\end{tabular}

Table.8 Relation between 6MWT (m) and TAPSE (mm)

\begin{tabular}{|l|l|l|}
\hline Stages & 6MWT $(\mathbf{m})$ & TAPSE $(\mathbf{m m})$ \\
\hline Stage-I & 431 & 17.43 \\
\hline Stage-II & 337.13 & 12.63 \\
\hline Stage-III & 222.15 & 7.27 \\
\hline Stage-IV & 165 & 3.73 \\
\hline
\end{tabular}


Table no. $7 \& 8$ shows that, with increasing GOLD stage the values of 6MWT, 2D FAC and TAPSE were decreasing.

The present study concluded that lower 2D FAC and TAPSE values were associated with higher GOLD staging. As higher GOLD staging is an indicator of adverse prognosis in patients of COPD, our study indirectly recognized adverse prognosis with lower 2D FAC and TAPSE values in COPD patients. Also our study showed that among 2D FAC and TAPSE, TAPSE was a more sensitive indicator of RV systolic dysfunction than 2D FAC, in GOLD stage-I \& II, however in stage-III \& IV both parameters had similar sensitivity i.e TAPSE was more sensitive in diagnosing mild RV dysfunction.

Our study also shown that with increasing GOLD stage, the values of 6MWT, 2D FAC and TAPSE were all decreasing i.e lower values of $2 \mathrm{D}$ FAC and TAPSE corresponded to lower values of FEV1 and 6MWT.

\section{Conclusion and Recommendations}

Our study concluded that higher GOLD stages of COPD correlated well with lower values of 2D FAC and TAPSE. Further our study also found that 2D FAC and TAPSE correlated well and equally with RV systolic dysfunction in the GOLD stages-III and IV, while in GOLD stages I and II, TAPSE was found to be more sensitive indicator of RV systolic dysfunction than 2D FAC i.e TAPSE was better parameter for diagnosing mild RV systolic dysfunction.

As right ventricular dysfunction and GOLD staging both have a prognostic importance, it is easy to easy to conclude the prognosis of COPD patients with help of TAPSE and 2D FAC because of inverse relationship of TAPSE and 2D FAC with GOLD stages of COPD. Also our study concluded that the lower values of $2 \mathrm{D}$ FAC and TAPSE correlated with lower values of 6MWT.

Therefore it is strongly recommended that 2D Echocardiography should be included in routine investigations of patients of COPD and all patients of COPD should be evaluated for RV systolic dysfunction using TAPSE and 2D FAC.

\section{References}

American Thoracic Society. 1995. Standardization of spirometry 1995 update. Am. J. Respire Crit. Care Med., 152: 1107-36.
Anavekar, N.S., Gerson, D., Skali, H., Kwong, R.Y., Yucerl, K., Solomon, S.D. 2007. Two-dimensional assessment of right ventricular function: an echocardiographic-MRI correlative study. Echocardiography, 24: 452-6.

Anconina, J., Danchin, N., Selton-Suty, C., Isaaz, K., Juilliere, Y., Buffet, P. et al. 1993. Non invasive estimation of right ventricular $\mathrm{dP} / \mathrm{dt}$ in patients with tricuspid valve regurgitation. Am. J. Cardiol., 71: 1495-7.

ATS statement. 2002. Guidelines for the six-minute walk test.

Brieke, A., DeNofrio, D. 2005. Right ventricular dysfunction in chronic dialated cardiomyopathy and heart failure. Coron Artery Dis., 16.5-11.

Brusasco, V., Crapo, R. 2005. Series 'ATS/ ERS Task Force' Standardization of lung function testing. Eur Respire J., 26(5): 319-38.

Chetan Rathi, Anil Wanjari, Sourya Acharya. 2015. Pulmonary Hypertension in Mild-Moderate COPD : An Early Link. J. Evidence based Med. Healthcare, Volume 2, Issue 42, Page: 7330-7340, DOI: 10.18410/jebmh/2015/991.

De groote, P., Millaire, A., Foucher-Hossein, C. 1998. Right ventricular ejection fraction is an independent predictor of survival in patients with moderate heart failure. J. Am. Coll. Cardiol., 32: 948-54.

Di salvo, T.G., mathier, M., semigran, M.J. 1995. Preserved right ventricular ejection fraction predicts exercise capacity and survival in advanced heart failure. J. Am. Coll. Cardiol., 25: 1143-53.

Gavazzi, A., Berzuini, C., Campana, C. 1997. Value of right ventricular ejection fraction in predicting short term prognosis of patients with severe chronic heart failure. J. Heart Lung Transplant, 16: 774-85.

Global initiative for chronic obstructive lung disease. Global strategy for this diagnosis, management and prevention of chronic obstructive lung disease. http://www.goldcopd.org/

Gupta, N.K., R.K. Agarwal, A.B. Srivastav. 2011. Echocardiographic evaluation of heart in chronic obstructive pulmonary disease patient and its corelation with the severity of disease. Lung India, 28(2): 105-9.

Jesper Kjaergaard, Kasper, K., Iversen, Dilek AKKan, Jacob Eifer Moller, Lars, V., Kober, Christian TorpPederson and Christian Hassager. 2009. Predictors of right ventricular function as measured by tricuspid annular plane systolic excursion in heart failure. Cardiovascular Ultrasound, 7: 51 doi: 1186/1476-7120-7-51. 
John, J., Reilly Edwin, K., Silverman Steven, D., Shapiro. Chronic Obstructive Pulmonary Disease: Introduction Harrison's Principles of Internal Medicine, 18e. Chapter 260 Page no. 2151-2159.

Maumita Das, Sumit Ray Tapadar, Anil Baran Singha Mahapatra, Shankar Paul Chowdhury, and Subrata Basu et al. 2014. Assessment of RV function in patients of COPD. J. Clin. Diagn. Res., 8(3): 11-13.

Rudski Lawrence, G., Lai Wyman, W., Afalilo Jonathan. 2010. Guidelines for the echocardiographic assessment of the right heart in adults: A report from the American Society of Echocardiography. $J$. Am. Soc. Echocardiogr., 23: 685-713.

Surendra, K., Sharma. Chronic Obstructive Pulmonary Disease. API textbook of medicine. 9th edition. Page no 1711-1718.

Ueti, O.M., Camargo, E.E., Ueti, A.A., Lima-Filho, E.C., Nogueira, E.A. 2002. Assessment of right ventricular function with Doppler echocardiographic indices derived from tricuspid annular motion: comparison with radionuclide angiography. Heart, 88: $244-8$.

\section{How to cite this article:}

Manish Kumar Bansal, Bechan Kumar Gautam. 2017. Comparative Analysis of Assessment of RV Dysfunction using 2D FAC and TAPSE in Patients of COPD and their Correlation with Pulmonary Function Test (PFT) and 6 Minute Walk Test (6MWT). Int.J.Curr.Res.Aca.Rev. 5(1), 54-60.

doi: http://dx.doi.org/10.20546/ijcrar.2017.501.006 